Baltic Astronomy, vol. 6, 637-650, 1997.

\title{
ON THE MANEV-TYPE TWO-BODY PROBLEM
}

Vasile Mioc ${ }^{1}$ and Cristina Stoica ${ }^{2}$

1 Astronomical Institute of the Romanian Academy, Astronomical Observatory Cluj-Napoca, Str. Ciresilor 19, RO-3400 Cluj-Napoca, Romania

2 Institute for Gravitation and Space Sciences, Gravitational Research Laboratory, Str. Mendeleev 21-25, RO-70168 Bucharest, Romania

Received March 20, 1997.

Abstract. The two-body problem in Manev-type fields (featured by potentials of the form $A / r+B / r^{2} ; r$ is the distance between particles, $A$ and $B$ are real parameters) constitutes a good model for various concrete physical problems of astronomy, astrophysics, relativity, atomic physics, mechanics, etc. We study relative motion in such fields both quantitatively and qualitatively. An analytic solution is obtained in a closed form. A qualitative investigation is performed, representing the motion in the $(1 / r, \dot{r})$ phase plane, where all the solutions are conic sections (or arcs of them). A bifurcation analysis is performed case by case for the whole allowed interplay among field parameters, angular momentum and total energy. Each solution is interpreted in terms of physical motion.

Key words: celestial mechanics: two-body problem - qualitative analysis

\section{INTRODUCTION}

The two-body problem with a quasihomogeneous potential of the form $A / r+B / r^{2}$ (where $r$ is the distance between particles, while $A$ and $B$ are real constants) is more than three centuries old. Newton was the first to consider it (for positive $A$ and $B$ ) in his Principia; in Book I, Article IX, Proposition XLIV, Theorem XIV, Corollary 2, he showed that such a force entails a precessionally elliptic relative orbit. In other words, the trajectory of a particle with respect to a 
fixed frame originated in the other particle will be an ellipse whose focal axis rotates in the plane of motion. Except for this result, Newton's research on such a model remained unpublished during his lifetime; however, the 1888-catalogue of the Portsmouth Collection of unpublished manuscripts points out his interest in this subject. The reason was the impossibility of explaining the Moon's apsidal motion within the framework of the inverse-square force model. After Newton, the $A / r+B / r^{2}$ potential was tackled by Clairaut, who finally abandoned it in favor of the classical potential.

One knows that the perihelion advance of the inner planets (especially that of Mercury) cannot be fully explained within the framework of the classical Newtonian law, even resorting to perturbation theory. The many pre- and post-relativistic laws (as those proposed by Hall and Newcomb, for instance) usually answered this question, but failed to explain other issues (as the secular motion of the Moon's perigee). As regards general relativity, it succeeded in explaining well such phenomena, both quantitatively and qualitatively. Unfortunately, this powerful theory, which answered many momentous questions in physics and astronomy, is not of much help for celestial mechanics. All attempts to formulate a meaningful relativistic $n$-body problem have failed to provide valuable results.

Therefore, the problem is to find a model able to respond to the theoretical needs of celestial mechanics (by keeping the simplicity and advantages of the Newtonian mechanics) and also to describe correctly the orbits coming close to collisions. In other words, we need a model able to maintain dynamical astronomy within the framework of classical mechanics, offering at the same time equally good justifications of the observed phenomena as in the relativity theory.

Such a model is that based on the above $A / r+B / r^{2}$ potential. Using physical principles, the Bulgarian physicist G. Manev (Maneff in his papers written in French or German) obtained a similar model in the twenties, and proposed it as an alternative substitute of relativity (Maneff 1924, 1925, 1930a,b). In the corresponding central force problem with unit mass for the "satellite" particle, Manev's potential gives $A=\mu, B=3 \mu^{2} /\left(2 c^{2}\right)$, where $\mu$ is the product between the Newtonian gravitational constant $G$ and the sum of the masses and $c$ is the speed of light. Fallen into oblivion for half a century, then pointed out by Hagihara (1975) as providing the same good theoretical approximations as the relativity theory (at the solar system level, at least), Manev's law was recently reconsidered 
in a series of studies having as a departure point the researches by Diacu (1993). For the two-body problem with this law, Mioc and Stoica (1995a,b) used Sundman-type transformations to regularize the motion equations and found a general solution of the regularized equations. Diacu et al. (1995) obtained an analytic solution and a local flow near collision. The isosceles three-body case was studied by Diacu (1993), while Ureche (1995) used this field to an astrophysical problem: the free-fall collapse of a homogeneous sphere.

The Manev potential for arbitrary positive values of $A$ and $B$ was also tackled. Lacomba et al. (1991) studied it in the Hamiltonian formalism for negative total energy; they also applied the KAM theory to a perturbed potential of this kind to prove a crucial result: if the motion equations undergo a slight perturbation, not necessarily Hamiltonian, most invariant cylinders and tori are topologically preserved. The Melnikov integral associated to the nonhyperbolic equilibria was computed by Casasayas et al. (1993). Aparicio and Floria (1996) showed that only the class of Hamiltonians to which Manev's model belongs admits linearization (reduction to harmonic oscillators). Diacu (1996) proved that Manev's case represents the only bifurcation of the flow among all quasihomogeneous potentials. Stoica and Mioc (1996a,b) depicted the problem geometrically in the usual phase planes, while Delgado et al. (1996) provided the complete analytic, geometric and physical description of the global flow in the space of McGehee's coordinates. The anisotropic Manev problem (important for understanding the connections between classical and quantum mechanics), suggested by Diacu (1993), was investigated by Craig et al. (1996). They obtained the local flow near collision, some elements of the global flow and the complete picture of the zero energy case.

One might say (and physicists do it often): to find the motion corresponding to the $A / r+B / r^{2}$ potential is an obsolete problem. Indeed, it appears (under different formulations) as an exercise in classical textbooks as those of Moulton (1923, p.96, Problem 4) or Goldstein (1980, p.123, Problem 14). Leaving aside the fact that the respective statement is incorrect in Goldstein's case or covers a very restricted area in Moulton's case, the above quoted results, and especially those obtained by Delgado et al. (1996), show how complex the problem is in reality.

The aim of this paper is to generalize the results given by Delgado et al. (1996), providing the complete analytic, geometric and physical description of the two-body problem associated to a Manev- 
type potential for any values (positive, negative or zero) of the field parameters $A$ and $B$.

The importance of such an analysis is emphasized by the multitude of concrete physical and astronomical situations modellable in connection with a potential of this kind. Some examples can be given. The motion in certain relativistic fields, truncating the negligible terms, is such a situation. The expressions correspond to Fock's field (Mioc 1994): $A=\left(2 E^{2}-1\right) \mu, B=3 \mu^{2} E^{2} / c^{2}$ (where $E=1+h / c^{2}$ and $h$ is the total energy per unit mass of the orbiting particle), hence $A>0, B>0$. For the relativistic field described by the Reissner-Nordström metric, we obtain $A>0, B<0$ as follows: $A=\mu, B=-G Q^{2} /\left(8 \pi \epsilon_{0} c^{2}\right)$, where $Q$ is the electric charge of the field-generating source, while $\epsilon_{0}$ is the electric permittivity of vacuum. A photogravitational field generated by a radiative source is featured by $A=\mu-\sigma L /(4 \pi m c$ ) (where $\sigma$ and $m$ are the crosssectional area and the mass of the orbiting body, while $L$ is the luminosity of the central body). In this case $B$ can be zero or nonzero when the gravitational component of the field is Newtonian or not. In its turn, $A$ can be positive, zero, or negative, as the Newtonian part of the gravitational force is stronger than, equal to, or weaker than the repulsive radiative force. Moreover, considering that $L$ is not constant (the case of a variable star for instance; see Saslaw 1978, Mioc \& Radu 1992, Selaru et al. 1993), we are in front of a perturbed Manev-type potential. The two-body problem with an equivalent gravitational parameter (Selaru et al. 1992) belongs to the same category, with $B=0$. For atomic physics, the potential energy of the outward electron in the field of the nucleus is given in a second approximation by a Manev-type model (Sommerfeld 1951, Belenkii 1981). Other implications in this direction were pointed out by Diacu (1993). For mechanics, the Manev-type model with $A=0$, $B>0$ has several interesting properties: it is a dividing line between two very different types of orbit behavior (McGehee 1981). Also in such a field, a system of $n$ particles moving along a straight line is completely integrable (Moser 1975); the time of total collision for the associated general $n$-body problem can be computed (Diacu 1990).

To end this series of examples we resort to some supplementary astronomical arguments. We saw that in Manev-type fields the natural, unperturbed motion of celestial bodies is precessional (as relativity had foreseen). In such fields the set of initial data leading to collisions has a positive measure (and in the solar system the collisions are not so rare as the Newtonian model asserts). Finally, we 
mention a scenario concerning the formation of a pulsar from two white dwarfs that spiral in toward each other, eventually merging into a single object (see Stephens 1996). Such a black hole effect (spiral collision) is possible in Manev's field (Diacu et al. 1995), but not in Newton's field.

In this paper we develop the Manev-type two-body problem, with interplay allowed among the field parameters, angular momentum and total energy. Reducing the framework to that of a central force problem, the analytic solution is obtained in closed form. Then a qualitative analysis is performed, based on the geometric representation of the motion in the $(1 / r, \dot{r})$-plane, where the corresponding trajectories are found to be only conic sections or arcs of them. Each allowed trajectory is interpreted in terms of physical motion, obtaining in this way an ample picture of the problem.

\section{ANALYTIC SOLUTION}

Consider hence the Manev-type two-body problem. We may reduce it to a central force problem (e.g. Arnold 1976) and study the motion of one body (of unit mass, hereafter particle) with respect to a fixed frame originated in the other body (hereafter center). This relative motion will be planar and described by the equation

$$
\ddot{\mathbf{r}}=-\frac{A}{r^{3}} \mathbf{r}-\frac{2 B}{r^{4}} \mathbf{r},
$$

where $\mathbf{r}=$ radius vector of the particle with respect to the center, $r=|\mathbf{r}|$ and dots mark time-differentiation.

Passing to polar coordinates $(r, u)$, equation (1) transforms into

$$
\begin{gathered}
\ddot{r}-r \dot{u}^{2}=-\frac{A}{r^{2}}-\frac{2 B}{r^{3}}, \\
r \ddot{u}+2 \dot{r} \dot{u}=0,
\end{gathered}
$$

system to which we attach the initial conditions

$$
(r, u, \dot{r}, \dot{u})\left(t_{0}\right)=\left(r_{0}, u_{0}, \dot{r}_{0}=V_{0} \cos \alpha, \dot{u}_{0}=V_{0} \sin \alpha / r_{0}\right),
$$

where $V_{0}=V\left(t_{0}\right), V=|\dot{\mathbf{r}}|$ (velocity), $\alpha=$ angle between initial radius vector and initial velocity.

The force field being central, the angular momentum is conserved, and (3) provides the first integral 


$$
r^{2} \dot{u}=C
$$

where $C=r_{0} V_{0} \sin \alpha$ is the constant angular momentum. The first integral of energy can also be easily obtained as

$$
V^{2} \equiv \dot{r}^{2}+r^{2} \dot{u}^{2}=\frac{2 A}{r}+\frac{2 B}{r^{2}}+h,
$$

where $h=V_{0}^{2}-2 A / r_{0}-2 B / r_{0}^{2}$ is the constant of energy.

The solution of the problem can be obtained in closed form. For instance, in the nonradial case $(C \neq 0)$, resorting to the usual technique (by (5), $d t=\left(r^{2} / C\right) d u$, and so forth), equation (2) leads to the Binet-type equation

$$
\frac{d^{2}(1 / r)}{d u^{2}}+\left(1-\frac{2 B}{C^{2}}\right)(1 / r)=\frac{A}{C^{2}}
$$

with the initial conditions (written in an equivalent form extracted from (4) and (5))

$$
\left(1 / r, \frac{d(1 / r)}{d u}\right)\left(u_{0}\right)=\left(1 / r_{0},-\dot{r}_{0} / C\right) .
$$

The general solution of the initial value problem attached to equation (7) depends on the sign of the parameter $\left(1-2 B / C^{2}\right)$ and will be for (a) $C^{2}<2 B$; (b) $C^{2}=2 B$; (c) $C^{2}>2 B$, respectively:

$$
\begin{aligned}
& r(u)=\left[\left(\frac{1}{r_{0}}+\frac{A}{2 B-C^{2}}\right) \tilde{C}(u)-\frac{\dot{r}_{0}}{\sqrt{2 B-C^{2}}} \tilde{S}(u)-\frac{A}{2 B-C^{2}}\right]^{-1} \\
& r(u)=\left[\frac{A}{2 C^{2}}\left(u-u_{0}\right)^{2}-\frac{\dot{r}_{0}}{C}\left(u-u_{0}\right)+\frac{1}{r_{0}}\right]^{-1} ; \\
& r(u)=\left[\left(\frac{1}{r_{0}}-\frac{A}{C^{2}-2 B}\right) \hat{C}(u)-\frac{\dot{r}_{0}}{\sqrt{C^{2}-2 B}} \hat{S}(u)+\frac{A}{C^{2}-2 B}\right]^{-1},
\end{aligned}
$$

where we abridged

$$
\begin{gathered}
(\tilde{S}, \tilde{C})(u)=(\sinh , \cosh )\left(\sqrt{1-2 B / C^{2}}\left(u-u_{0}\right)\right) \\
(\hat{S}, \hat{C})(u)=(\sin , \cos )\left(\sqrt{2 B / C^{2}-1}\left(u-u_{0}\right)\right)
\end{gathered}
$$


Formulae (8) were actually obtained (in a slightly modified form) by Delgado et al. (1996). Although the respective paper referred to the Manev problem with $A$ and $B$ as positive constants, the analytic form of the solutions remains valid for any real value of $A$ and $B$ (and for $C \neq 0$, of course).

The radial case $(C=0)$ can also be solved, by resorting to equation (6) with $\dot{u}=0$. We shall not dwell upon it for two reasons. On the one hand, the analytic solution of the initial value problem attached to Eq. (6) (in which $\dot{u}=0$ ) will be of the form $t=t(r)$, relation invertible only in particular cases. On the other hand, we are more interested in a qualitative investigation of the particle behavior. The analysis we are going to perform in the next section covers both cases.

\section{GEOMETRIC DESCRIPTION}

To study the particle behavior from a qualitative standpoint, we eliminate $\dot{u}$ between (5) and (6), obtaining

$$
\frac{C^{2}-2 B}{r^{2}}-\frac{2 A}{r}+\dot{r}^{2}-h=0 \text {. }
$$

This represents in the $(1 / r, \dot{r})$-plane a family of conic sections whose kind (ellipses, parabolas, hyperbolas) and nature (nondegenerate, degenerate) are respectively given by the parameters $\delta=C^{2}-2 B$ and $\Delta=h\left(2 B-C^{2}\right)-A^{2}$. Observe that there exists a critical energy level

$$
h_{c}=\frac{A^{2}}{2 B-C^{2}}
$$

for which $\Delta=0$ (degenerate conic sections).

If $C^{2}<2 B(\delta<0)$, Eq. (9) represents a family of hyperbolas (Fig. 1) centered in $\mathrm{P}\left(-A /\left(2 B-C^{2}\right), 0\right)$. For $h<h_{c}$ the semiaxes are $\sqrt{\left(h_{c}-h\right) /\left(2 B-C^{2}\right)}$ and $\sqrt{h_{c}-h}$, while the foci are lying on the $1 / r$-axis. For $h=h_{c}$, Eq. (9) represents the respective asymptotes and for $h>h_{c}$ it describes the family of conjugate hyperbolas.

If $C^{2}=2 B(\delta=0)$, Eq. (9) represents a family of parabolas, nondegenerate for $A \neq 0$ (Figs. 2a, 2c). For $A=0(\Delta=0)$, every parabola degenerates into a pair of lines (distinct or not) parallel to the $1 / r$-axis (Fig. 2 b).

If $C^{2}>2 B(\delta>0)$, Eq. (9) represents a family of ellipses (Fig. 3) with the same center and semiaxes as the above hyperbolas. The 


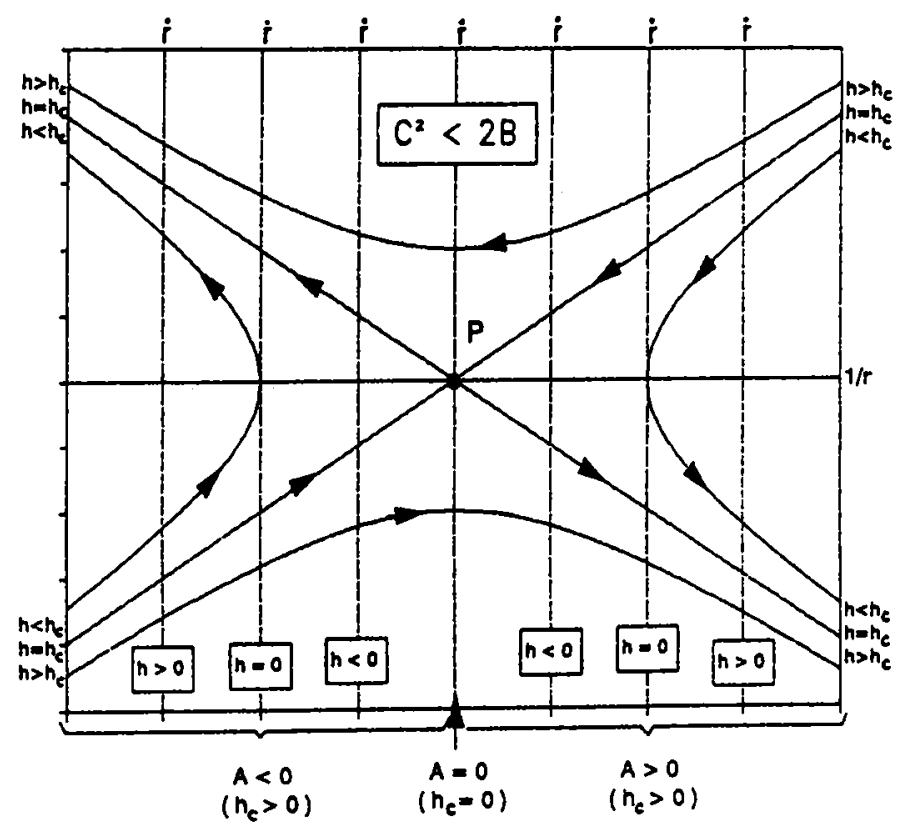

Fig. 1. The flow for $C^{2}<2 B$.

ellipses are real for $h>h_{c}(\Delta<0)$, they reduce to the center $\mathrm{P}$ of the family if $h=h_{c}$ and they are imaginary if $h<h_{c}$.

Knowing that only the curves lying in the halfplane $1 / r>0$ represent real motion in the physical plane, and using (9), we can remove the forbidden combinations $\{A, B, C, h\}$, which lead to impossible real motion and geometrically mean:

$\left\{C^{2}=2 B, A<0, h \leq 0\right\}$ : parabolas lying wholly in the zone $1 / r \leq 0$

$\left\{C^{2}=2 B, A=0, h<0\right\}$ : pairs of imaginary parallel lines;

$\left\{C^{2}>2 B, A \leq 0, h \leq 0\right\}$ : real ellipses lying wholly in the zone $1 / r \leq 0$

$\left\{C^{2}>2 B, A>0, h<h_{c}\right\}:$ imaginary ellipses.

The trajectories in the $(1 / r, \dot{r})$-plane are represented in Figs. 1 $\left(C^{2}<2 B\right), 2\left(C^{2}=2 B\right)$ and $3\left(C^{2}>2 B\right)$. The corresponding curves (arcs of curves) for $1 / r>0$ and for each allowed combination $\{A, B, C, h\}$ are easy to identify.

There are equilibria for each case: $\mathrm{P}$ is a saddle point in Fig. 1 and a center in Fig. 3 ; in Fig. $2 b$ the whole semiaxis $(1 / r>0, \dot{r}=0)$ consists of stable equilibria. 

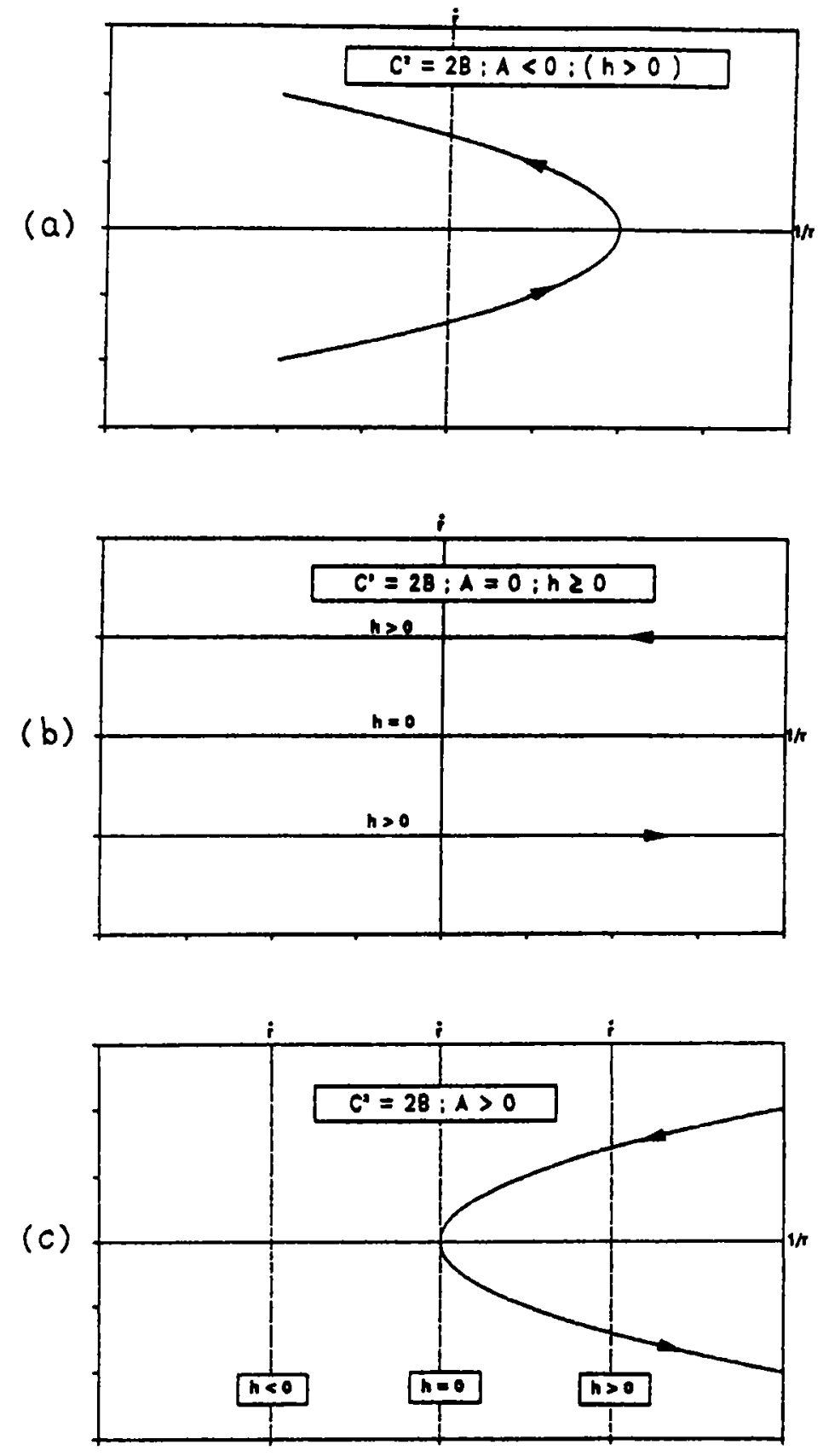

Fig. 2. The flow for $C^{2}=2 B$ and (a) $A<0$, (b) $A=0$, (c) $A>0$. 


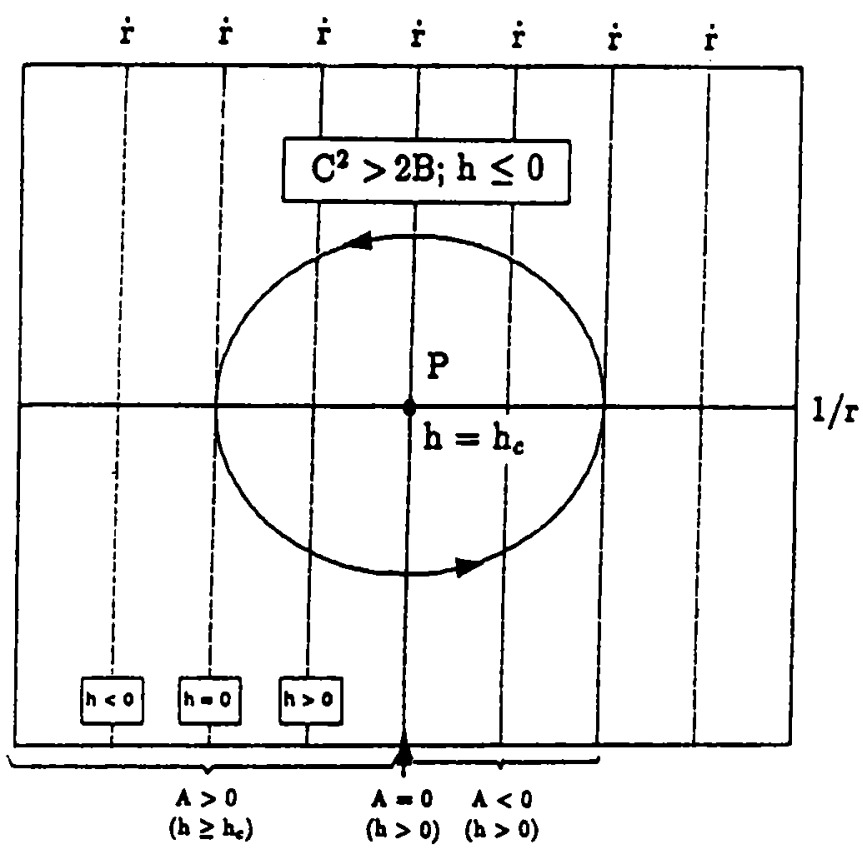

Fig. 3. The flow for $C^{2}>2 B$.

To end this section, we would like to emphasize the main advantage offered by the use of the $(1 / r, \dot{r})$-plane in describing the problem geometrically. The trajectories in this plane are conic sections, whose features are very well known (providing immediately the qualitative behavior of the particle and facilitating the physical interpretation), while the usual $(r, \dot{r})$ phase curves are more complicated (see Stoica $\&$ Mioc 1996a,b). Also, if we choose the velocity plane $\left(V_{u}=C / r\right.$, $\left.V_{r}=\dot{r}\right)$, the trajectories are found again to be conic sections, but the rectilinear case cannot be studied in such a way.

\section{PHYSICAL INTERPRETATION}

First of all, let us clear up the nature of the physical motion represented geometrically in Figs. 1-3. By (5), $u$ varies monotonically $(C \neq 0)$ or remains constant $(C=0)$ all along the motion. Consequently, every trajectory segment in the upper/lower halfplane of figures physically means spiral $(C \neq 0)$ or rectilinear $(C=0)$ motion performed outwards/inwards. 
Tables 1-3 (each one interpreting the corresponding figure) synthesize the particle behavior in terms of physical motion for the whole allowed interplay among field parameters, angular momentum and total energy. The symbols used in tables are:

$0 \rightarrow 0$ : orbits ejecting from collision and then tending back to collision (the particle cannot escape);

$0 \rightarrow \infty$ : orbits ejecting from collision and then tending to infinity (after ejection the particle cannot collide anymore with the center);

$\infty \rightarrow 0$ : orbits coming from infinity and tending to collision (no escape is possible);

$\infty \rightarrow \infty$ : orbits coming from infinity and then tending back to infinity (no collision is possible);

SE: stable equilibrium orbits (circular for $C \neq 0$, or stable rest for $C=0$ );

UE: unstable equilibrium orbits (circles or rest as $C \neq 0$ or for $C=0$, respectively).

To have a more detailed picture of the physical motion, some explanatory notes are necessary: if $C=0$, the collision (ejection) is rectilinear; if $C \neq 0$, the situation changes: the particle spirals around the center, performing infinitely many rotations immediately before collision (after ejection) (cf. Delgado et al. 1996). The velocity has an infinite value in these cases, except for $\left\{C^{2}=2 B, A=0, h>0\right\}$ (Table 2, Fig. 2b), when $V=\sqrt{h}$ all along the motion.

When the particle escapes to infinity, its velocity tends asymptotically (except the above constant $V$ case) to $\sqrt{h}$ by lower values if $A<0$ or $\left\{C^{2}>2 B, A=0\right\}$ or by higher values if $A>0$ or $\left\{C^{2}<2 B, A=0\right\}$.

The case $\left\{C^{2}>2 B, A>0, h_{c}<h<0\right\}$ means motion with periodic character; neither collision nor escape are possible. In rectilinear motion $(C=0)$ the particle librates radially $\left(0<r_{\min } \leq r \leq\right.$ $\left.r_{\max }<\infty\right)$. For $C \neq 0$ the orbits are precessional ellipses (cf. Diacu et al. 1995) inside an annulus: the motion is periodic (closed curves) if $\sqrt{1-2 B / C^{2}}$ is rational (see the last formula (8)), or quasiperiodic (unclosed curves filling densely an annulus) if $\sqrt{1-2 B / C^{2}}$ is irrational (see Arnold 1976, Delgado et al. 1996).

Finally, one can see that for rectilinear motion $(C=0)$ all cases concerning comparison between $C^{2}$ and $2 B$ reduce to cases concerning the sign of $B$. In such a situation the column $A=0$ in Table 2 corresponds to motion (or rest) in the absence of the field. 
Table 1. $C^{2}<2 B ; h_{c} \geq 0$.

\begin{tabular}{|c|c|c|c|}
\hline $\bar{h}$ & $A<0$ & $A=0\left(h_{c}=0\right)$ & $\overline{A>0}$ \\
\hline$h<0$ & $0 \rightarrow 0$ & $0 \rightarrow 0$ & $0 \rightarrow 0$ \\
\hline$h=0$ & $0 \rightarrow 0$ & \multirow{3}{*}{$0 \rightarrow \infty, \infty \rightarrow 0$} & $0 \rightarrow \infty, \infty \rightarrow 0$ \\
\hline $0<h<h_{c}$ & $0 \rightarrow 0, \infty \rightarrow \infty$ & & $0 \rightarrow \infty, \infty \rightarrow 0$ \\
\hline$h=h_{c}$ & $\begin{array}{c}\mathrm{u} \rightarrow \mathrm{UE}, \infty \rightarrow \mathrm{UE} \\
\mathrm{UE} \rightarrow 0, \mathrm{UE} \rightarrow \infty \\
\mathrm{UE}\end{array}$ & & $0 \rightarrow \infty, \infty \rightarrow 0$ \\
\hline$h>h_{c}$ & $0 \rightarrow \infty, \infty \rightarrow 0$ & $0 \rightarrow \infty, \infty \rightarrow 0$ & $0 \rightarrow \infty, \infty \rightarrow 0$ \\
\hline
\end{tabular}

Table 2. $C^{2}=2 B$.

\begin{tabular}{|c|c|c|c|}
\hline \hline$h$ & $A<0(h>0)$ & $A=0(h \geq 0)$ & $A>0$ \\
\hline \hline$h<0$ & - & - & $0 \rightarrow 0$ \\
\hline$h=0$ & - & SE & $0 \rightarrow \infty, \infty \rightarrow 0$ \\
\hline$h>0$ & $\infty \rightarrow \infty$ & $0 \rightarrow \infty, \infty \rightarrow 0$ & $0 \rightarrow \infty, \infty \rightarrow 0$ \\
\hline \hline
\end{tabular}

Table 3. $C^{2}>2 B ; h_{c} \leq 0$.

\begin{tabular}{|c|c|c|c|}
\hline \hline$h$ & $A<0(h>0)$ & $A=0(h>0)$ & $A>0\left(h \geq h_{c}\right)$ \\
\hline \hline$h=h_{c}$ & - & - & SE \\
\hline$h_{c}<h<0$ & - & - & $\begin{array}{c}\text { quasiperiodic, } \\
\text { periodic }\end{array}$ \\
\hline$h=0$ & - & - & $\infty \rightarrow \infty$ \\
\hline$h>0$ & $\infty \rightarrow \infty$ & $\infty \rightarrow \infty$ & $\infty \rightarrow \infty$ \\
\hline
\end{tabular}

\section{REFERENCES}

Aparicio I., Floria L. 1996, On Perturbed Two-Body Problems and Harmonic Oscillators, C. R. Acad. Sci. Paris, 323, sér. IIb, 71 
Arnold V. 1976, Les méthodes mathématiques de la mécanique classique, Mir Publishing House, Moscow

Belenkii I. M. 1981, A Method of Regularizing the Equations of Motion in the Central Force-Field, Celest. Mech. 23, 9

Casasayas J., Fontich E., Nunes A. 1993, Transversal Homoclinic Orbits for a Class of Hamiltonian Systems, in Lacomba E. \& Llibre J. (eds), Hamiltonian Systems and Celestial Mechanics, Advanced Series in Nonlinear Dynamics, Vol.4, World Scientific, Singapore, p. 35

Craig S., Diacu F. N., Lacomba E. A., Perez E. 1996. On the Anisotropic Manev Problem, Preprint DMS-736-IR, University of Victoria, Canada Delgado J., Diacu F. N., Lacomba E.A., Mingarelli A., Mioc V., Perez E., Stoica C. 1996, The Global Flow of the Manev Problem, J. Math. Phys., 37, 2748

Diacu F. N. 1990, Total Collapse Dynamics for Particle Systems, Libertas Math., 10, 161

Diacu F. N. 1993, The Planar Isosceles Problem for Maneff's Gravitational Law, J. Math. Phys., 34, 5671

Diacu F.N. 1996, Near-Collision Dynamics for Particle Systems with Quasihomogeneous Potentials, J. Diff. Eq., 128, 58

Diacu F. N., Mingarelli A., Mioc V., Stoica.C. 1995, The Manev TwoBody Problem: Quantitative and Qualitative Theory, in Agarwal R.P. (ed.), Dynamical Systems and Applications, World Scientific Series in Applicable Analysis, Vol. 4, World Scientific, Singapore, p. 213

Goldstein H. 1980, Classical Mechanics, 2nd revised ed., Addison-Wesley, Reading, Massachusetts

Hagihara Y. 1975, Celestial Mechanics, Vol.2, Part 1, The MIT Press, Cambridge, Massachusetts

Lacomba E. A., Llibre J., Nunes A. 1991, Invariant Tori and Cylinders for a Class of Perturbed Hamiltonian Systems, in Ratiu T. (ed.), The Geometry of Hamiltonian Systems, Proceedings of a Workshop, June 5-16, 1989, Springer-Verlag, New York, p. 375

McGehee R. 1981, Double Collisions for a Classical Particle System with Nongravitational Interactions, Comment. Math. Helvetici, 56, 524

Maneff G. 1924, La gravitation et le principe de l'égalité de l'action et de la réaction, C. R. Acad. Sci. Paris, 178, 2159

Maneff G. 1925, Die Gravitation und das Prinzip von Wirkung und Gegenwirkung, Z. Phys., 31, 786

Maneff G. 1930a, Le principe de la moindre action et la gravitation, C. R. Acad. Sci. Paris, 190, 963

Maneff G. 1930b, La gravitation et l'énergie au zéro, C. R. Acad. Sci. Paris, 190, 1374 
Mioc V. 1994, Elliptic-Type Motion in Fock's Gravitational Field, Astron. Nachrichten, 315, 175

Mioc V., Radu E. 1992, Orbits in an Anisotropic Radiation Field, Astron. Nachrichten, 313, 353

Mioc V., Stoica C. 1995a, Discussion et résolution complète du problème des deux corps dans le champ gravitationnel de Maneff, C. R. Acad. Sci. Paris, 320, sér. I, 645

Mioc V., Stoica C. 1995b, Discussion et résolution complète du problème des deux corps dans le champ gravitationnel de Maneff (II), C. R. Acad. Sci. Paris, 321, sér. I, 961

Moser J. 1975, Three Integrable Hamiltonian Systems Connected with Isospectral Deformation, Adv. Math., 16, 197

Moulton F.R. 1923, An Introduction to Celestial Mechanics, 2nd ed., Macmillan, London

Saslaw W.C. 1978, Motion around a Source whose Luminosity Changes, ApJ, 226, 240

Sommerfeld A. 1951, Atombau und Spektrallinien, Bd. 1, Braunschweig Stephens S. 1996, Second-Chance Planets, Astronomy, Vol.24, No. 1, 50 Stoica C., Mioc V. 1996a, A Qualitative Study of the Two-Body Problem in Maneff-Type Fields, Rom. Astron. J., 6, 81

Stoica C., Mioc V. 1996b, On the Two-Body Problem in Maneff-Type Fields, Bull. Astron. Obs. Belgrade, No. 154, 1

Selaru D., Cucu-Dumitrescu C., Mioc V. 1992, On a Two-Body Problem with Periodically Changing Equivalent Gravitational Parameter, Astron. Nachrichten, 313, 257

Selaru D., Cucu-Dumitrescu C., Mioc V. 1993, Periodic Motions around Pulsating Stars, Ap\&SS, 202, 11

Ureche V. 1995, Free-Fall Collapse of a Homogeneous Sphere in Maneff's Gravitational Field, Rom. Astron. J., 5, 145 